\title{
AN AEROSOL LIDAR DATA VISUALIZATION METHOD BASED ON ARCGIS
}

\author{
Chang Shu ${ }^{1}$, Hu Zhao ${ }^{2, *}$, Hailun Zhang ${ }^{2}$, Xingkai Wang ${ }^{1}$, Yapeng Liu ${ }^{1}$ \\ ${ }^{1}$ School of Computer Science and Engineering, North MinZu University Yinchuan 750021, China - (410513982, 1395585521, \\ 982843635)@qq.com \\ ${ }^{2}$ School of Electrical and Information Engineering, North MinZu University Yinchuan 750021, China - zhaoh_1@ yeah.net, \\ 809361497@qq.com
}

\section{Commission III, WG III/8}

KEY WORDS: Extinction Coefficient, ArcGIS, Natural Neighborhood Method, Lidar, Aerosol

\begin{abstract}
:
The geographical information, temporal and spatial distribution of pollution sources cannot be accurately described by the traditional aerosol lidar inversion data. In order to accurately locate the pollution sources and monitor the spatial and temporal distribution of pollutants, an aerosol lidar data visualization method based on ArcGIS is proposed in this paper. A natural neighborhood method is used to perform group interpolation on ArcGIS software, and the range-extinction coefficient relationship diagram is plotted using the aerosol optical data obtained from Klett inversion algorithm. The geographic information is added to the lidar inversion data in this diagram. The aerosol spatial and temporal distribution range and the direction of aerosol diffusion are accurately displayed directly. The change of aerosol concentration is plotted in different colors, the visually displaying the concentration of pollutants in the detection area is realized. The aerosol detection data in the sunny and haze days in Yinchuan are visualized in this paper. The result shows that this method can quickly determine the geographical location of pollution sources and visualize the spatial and temporal distribution of aerosols. This method can be used to display aerosol data with geographic information for meteorological and environmental protection departments. This method can provide data support for meteorological and environmental protection departments to quickly determine the location and concentration of pollution sources.
\end{abstract}

\section{INTRODUCTION}

In atmospheric science, the system composed of atmospheric medium and suspended solid or liquid particles with size ranging from 0.001 to 100 , such as dust, smoke, ice crystal and pollen mixed in it, is called aerosol. Aerosols are mainly distributed in the troposphere and affect the balance of the atmosphere-earth system through direct and indirect radiation forcing. First, the direct radiation forcing effect of aerosols on the warming and cooling of the climate affects the temperature change. Second, aerosol indirect radiation forcing plays an important role in cloud physical characteristics, reflectivity and cloud cover, and prolonging cloud life cycle, thus affecting precipitation. Third, various aerosol particles produce chemical changes in the atmosphere, which affects the proportion of nitrate, ozone, sulfate and other elements in the air. Research on particulate pollutants in the atmosphere plays a crucial role in human health, environment and global climate change and other related research fields.

At present, the visibility instruments and hygrometers are used in the near-ground sounding network include aerosol particle size spectrometer and solar photometer, etc. In high space, remote sensing satellites are mainly used for atmospheric environment monitoring. However, the current near-ground detection equipment has the shortcomings of harsh operating conditions, low measurement accuracy and poor stability, while remote sensing satellites are expensive and not convenient to use. Aerosol lidar is a remote sensing detection instrument integrating photoelectric detection and weak radar signal processing technology with laser as the light source. It is a powerful tool for real-time monitoring of atmospheric aerosols and clouds with high spatial and temporal resolution, and studying their vertical distribution, composition and optical thickness. It has wide spectral range and it can obtain rich atmospheric optical parameters. Therefore, it becomes the mainstream technology of atmospheric remote sensing. In the actual detection, according to the working principle, the lidar is divided into Mie scattering lidar for measurement of aerosol and cloud composition. Doppler lidar for measuring wind speed and direction of the wind profile, Raman scattering lidar for measuring trace gases, water vapor composition, and the lidar for monitoring $\mathrm{O}_{3}$ and $\mathrm{CO}_{2}$ etc.

In the last three decades, lidar technology has developed rapidly. Althausen et al. (1992) constructed a 6-wavelength, 11-channel scanning Raman aerosol lidar in troposphere research institute, university of Leipzig, Germany. Goldsmith et al. (1998) developed a Raman lidar system to retrieve the profiles of atmospheric water vapor, clouds and aerosols, and measured the characteristics of water vapor and aerosols with a lidar at wavelength of $355 \mathrm{~nm}$. Muller et al. (1999) successively designed a movable 6-wavelength 11-channel multi-wavelength Raman lidar system composed of two Nd:YAG lasers and two dye lasers. Hong et al. (2004) from GIST, South Korea developed a multi-wavelength lidar composed of 355, 532 and $1064 \mathrm{~nm}$ wavelengths for detecting and retrieving aerosol optical and physical parameters. Hamed Parsiani et al. (2008) used multi-wavelength lidar of the city university of New York to retrieve aerosol optical and physical parameters such as

\footnotetext{
* Corresponding author: Hu Zhao - zhaoh_1@ @yeah. net
} 
aerosol backscattering coefficient, extinction coefficient, optical thickness and particle spectral distribution. In the past 20 years, lidar technology has also been developed gradually in China. Zhou et al. (2000) developed a dual-wavelength Mie scattering lidar, which is used for long-term detection of vertical profile of tropospheric aerosol extinction coefficient at $532 \mathrm{~nm}$ and 1065 $\mathrm{nm}$. Zhong et al. (2008) developed two-field Mie scattering lidar, which realized simultaneous detection of high and low layers of atmosphere. Liu et al. (2013) developed a three-wavelength Raman polarization lidar, which was used to study the microphysical properties of aerosols and the physical properties of cirrus clouds.

However, the scatter diagram, broken line diagram and THI diagram are generally drawn after the data processing of lidar detection at present. The temporal and spatial evolution process of aerosols cannot be directly shown on the map. Aerosol lidar technology integrated ArcGIS has advantages in data visualization, information operations, and network sharing. It can realize the effective information collection, processing and decision support. In this paper, the graphical display of pollutant concentration monitoring is realized intuitively on the map. It displays the scope, trend, and the numerical value in different colors using ArcGIS interpolated by natural neighborhood method. This technology becomes the breakthrough and innovation point of current pollutant concentration monitoring. The first purpose of this study is to provide decision-making assistance for meteorological bureaus and environmental protection departments at all levels. Second, the multi-level pollution of atmospheric environment information is released to public.

\section{COMPOSITION AND PRINCIPLE OF AEROSOL LIDAR}

\subsection{Composition and Measurement of Aerosol Lidar}

Aerosol lidar system is mainly composed of laser pulse emission system, optical receiving system, spectral system, photoelectric detection unit and signal acquisition system, as shown in Figure 1. The laser pulse emission system is composed of Nd:YAG solid laser (model SGR-10), SHG, THG, and repeated emission of $355 \mathrm{~nm}, 532 \mathrm{~nm}$ and $1064 \mathrm{~nm}$ laser beams at $10 \mathrm{~Hz}$. The optical receiving system adopts cassegrain refracted telescope to receive backscattered light. The optical signals processed by the spectroscopic system are converted into electrical signals by three PMT (Photo Multiplier Tube) and an APD (Avalanche Photo Diode). After signal amplification by micro-current operation amplifier circuit, data acquisition is carried out by the signal acquisition system.

Scanning measurement of the aerosol lidar is carried out by means of rotating platform. The sampling rate was $100 \mathrm{M} / \mathrm{s}$, the cumulative time of each detection was $10 \mathrm{~min}$, the recording length was 10,000 points, and the average time was 5,000 times. Multiple sets of data are measured at each wavelength at different angles.

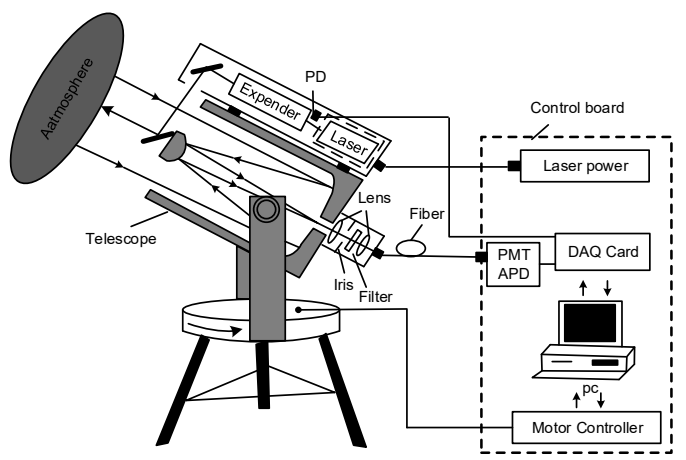

Figure 1. Composition diagram of aerosol lidar

\subsection{Klett Inversion Algorithm}

Generally, the lidar equation is:

$$
P(R)=P_{0} \eta_{0} Y\left(R_{0}\right) \frac{c t_{p}}{2} A_{R} \frac{\beta(R)}{R^{2}} \exp \left\lfloor-2 \int_{0}^{R_{0}} \alpha(r) d r\right\rfloor
$$

Where $P(R)$ is the signal intensity, $P_{0}$ is laser emission intensity, $\eta_{0}$ is the total efficiency of the optical element, $Y\left(R_{0}\right)$ is the geometric overlap factor, $\mathrm{c}$ is the speed of light, $t_{p}$ as the laser pulse width, $A_{R}$ is the aperture area, $\beta(R)$ is the backscatter coefficient, $\alpha(r)$ is extinction coefficient.

Let $\mathrm{S}(R)=\operatorname{In}\left[P(R) R^{2}\right]$, then we can get:

$$
\mathrm{S}(R)-S\left(R_{0}\right)=\ln \frac{\beta(R)}{\beta\left(R_{0}\right)}-2 \int_{R_{0}}^{R} \alpha(r) d r
$$

Assuming that atmospheric aerosols are uniformly distributed, then $\frac{d \beta}{d R}=0$, and then the extinction coefficient of aerosols is:

$$
\alpha(r)=-\frac{d S}{2 d R}
$$

It is assumed that the relationship between backscattering coefficient and extinction coefficient is as follows:

$$
\beta(R)=C_{0} \alpha(R)^{k}
$$

In the this equation, $C_{0}$ is a constant, and the value of $k$ is determined by the wavelength of lidar emission beam and aerosol types, and the value range is $0.67 \sim 1.0$. Assuming that $k$ is a constant, let $R \leq R_{m}$, then the extinction coefficient at range $R$ can be expressed as:

$$
\mathrm{a}(R)=\frac{e\left\{\frac{\left[S(R)-S\left(R_{m}\right)\right]}{k}\right\}}{\mathrm{a}\left(R_{m}\right)^{(-1)}+\frac{2}{k} \int_{R}^{R m} e\left\{\frac{\left[S(\mathrm{r})-S\left(R_{m}\right)\right]}{k}\right\} d r}
$$

$\alpha\left(R_{0}\right)$ can be calculated through:

$$
\alpha\left(R_{0}\right) \approx \frac{S\left(R_{0}\right)-S\left(R_{m}\right)}{2\left(R_{m}-R_{0}\right)}
$$


According to the formula, the extinction coefficient of atmosphere below the height of $R_{m}$ can be calculated.

\section{NATURAL NEIGHBORHOOD METHOD IS USED FOR INTERPOLATION IN ARCGIS}

Although the types of visualizations data are various, the distribution and connectivity of the data vary greatly, but the basic flow of data visualizations is the same. It can be shown in Figure 2.

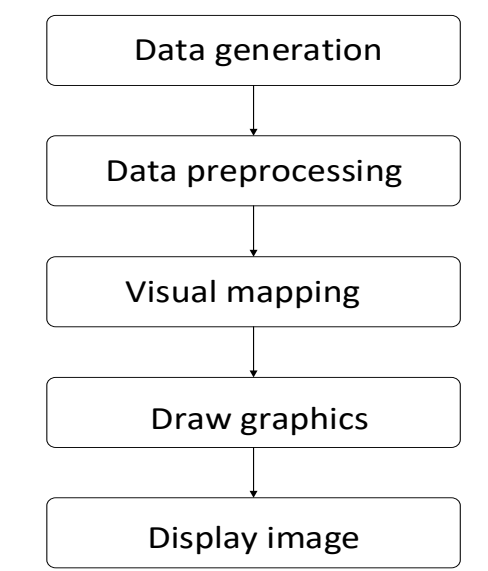

Figure 2. Visualization basic flow chart

Experimental measurement or computer numerical simulation is used to generate experimental data which is data generation. Data processing is mainly composed of data filtering and data transform, it eliminates noise from original data and transform original data into a form suitable for subsequent visualization. The common data processing method includes gradient calculation, noise elimination and data transformation.

Visual mapping is the core of the whole process. Its meaning is the transformation of processed raw data into set pixels and attributes that can be drawn. The meaning of "mapping" includes the design of visualization scheme, and it is urgent to decide what can be seen in the final generated image and how to present it. That is, using brightness, shape, color, and other properties to represent the properties and characteristics of the original data. Different visualization technologies are adopted according to different application fields and data types.

The mapping image converts the above generated pixels and attributes into images that can be displayed. The related technologies of computer graphics are applied, including visual transformation, illumination calculation, hidden face elimination and scanning transformation. Graphics hardware or graphics software packages can also be used to complete the above functions.

Image transformation display includes image geometry transformation, image compression, color quantization, image format conversion and image dynamic output.

\subsection{Data Management}

Shapefle is a kind of vector data format provided by ESRI. A Shapefile consists of a series of files, among which the main basic files are coordinate files (.shp), index files (.shx) and properties files (.dbf). The coordinate file is used to record the spatial coordinate information. The index file contains the index information of the coordinate file, where each record contains the corresponding coordinate file and records the offset of the header file of the distance coordinate file. Property file is a standard DBF file, which is used to record the property information of the file. According to the longitude and latitude of the observation points, a series of coordinate points are generated and stored as Shapefile file, so as to achieve linear interpolation on the map.

\subsection{Natural Neighborhood Interpolation}

Color mapping method is a common visual expression method in the description of atmospheric parameters. The main technology of color mapping method is that the numerical point $f(x, y)$ of different atmospheric parameters in lidar is represented with the corresponding color value color $(x, y)$. By comparing the color bar value, researchers can see the distribution of atmospheric parameters through color and brightness of image. The maximum $v_{\max }$ and minimum $v_{\min }$ of atmospheric parameters can be found from the data of atmospheric parameters in this color mapping. Moreover, according to the difference value $\left(v_{\max }-v_{\min }\right)$, several atmospheric regions were divided, namely, color labeling was carried out. The continuous extinction coefficient of atmosphere was mapped into the hue of stair jumping. The smooth natural neighborhood interpolation method was used to calculate the corresponding color value of atmospheric parameters in the cutting plane.

The interpolation data are all discretized data obtained by aerosol lidar sampling. If the data is drawn directly, the generated image will be blurred and the original information will be lost. Interpolation can predict pixel values in the grid based on limited sample data, and it can predict unknown values of geographical point data. Interpolation technique is used to represent the approximate value distribution of data between voxels or faults. So the interpolation is used to reconstruct the discrete data field.

The interpolation methods commonly used in the rendering of image are Kriging method, inverse distance weight method and natural neighborhood method. Kriging interpolation method has complicated calculation steps and slow interpolation speed. The inverse distance weight method is sensitive to the power selection of the weight function. It is easily affected by the sampling cluster, and it results in the "Bovine Eye " effect. Compared with other interpolation methods, Natural neighborhood method can find the set of discrete points closest to the interpolation points. These discrete points are interpolated according to the weight and the scale of the size of the region. Weights are allocated according to the overlap ratio of the nearest neighbor polygon of two discrete points and the Voronoi polygon formed by interpolation points of surrounding sampling points.

All interpolation points are related to the neighboring Voronoi polygon. The initial Voronoi polygon is composed of the specified set of points, and the new Voronoi polygon will be constructed at the interpolation points. The overlap ratio between the constructed polygon and the original polygon will be used as the weight factor, as shown in Figure 3. The surface will pass through the input sample and be smooth in all locations except the input sample position. In contrast, a range-based interpolator assigns the same weight to the northernmost and northeastern points based on the same distance from the interpolation points. Natural neighborhood method will assign 
weights of $19.12 \%$ and $0.38 \%$ respectively according to the overlap percentage, and thus generated results are more accurate. Considering the time complexity of the atmospheric parametersand the image algorithm, on the premise of ensuring the image quality, Natural neighborhood method is adopted for interpolation.

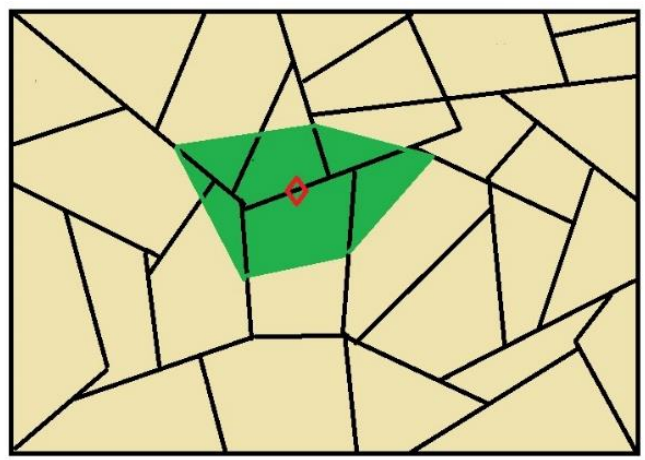

Figure3. Create Voronoi polygons around interpolation points

\section{EXPERIMENTAL RESULTS AND ANALYSIS}

The experiment was conducted at North MinZu University $\left(106.26^{\circ} \mathrm{E}, 38.46^{\circ} \mathrm{N}, 1100 \mathrm{~m}\right.$ above sea level ) in XiXia district of YinChuan city from March 11 to 17, 2018. The horizontal and slant scanning are conducted to monitor pollution sources and local aerosol. The spatial and temporal distributions of local aerosol are plotted.

\subsection{Rapid Identification of Pollution Sources by Horizontal Scanning}

With the help of aerosol lidar, we conducted the monitoring of aerosols at sunny day and haze weather. The horizontal scanning was centred on North MinZu University, and the farthest distance was 12,750 meters.

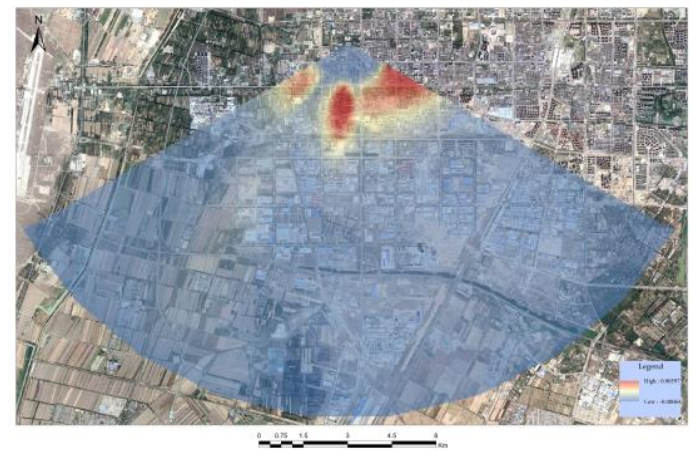

Figure 4. Horizontal scanning results of sunny days

Figure 4 shows the observations at 19:20 on March 11, 2018. It can be clearly seen from the figure that there are obvious red patchy pollution sources around 450 meters south of the instrument placement point. Through field investigation, the scene is a local food street, where a large number of aerosol particles generated by outdoor barbecues. The aerosol particles float into the air and spread around, the pollution sources in the area are detected.

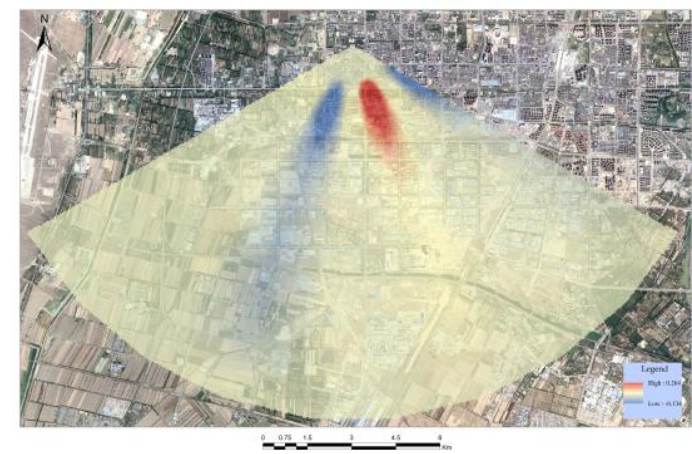

Figure 5. Horizontal scanning results of haze weather

Figure 5 shows the observations at 21:30 on March 13, 2018. The weather was moderate haze, which was significantly different from sunny weather. It can be seen from the figure that the overall color of the area is darker than sunny day, and there are small pieces of red patchy pollution sources. It means that the concentration of aerosol particles is large on that day. Aerosol particles are distributed over a wide range.

\subsection{Slant Scanning to Detect Spatial and Temporal Distribution of Aerosols}

Combined with the aerosol lidar slant detection, the observation of the stationary point was conducted in Yinchuan.

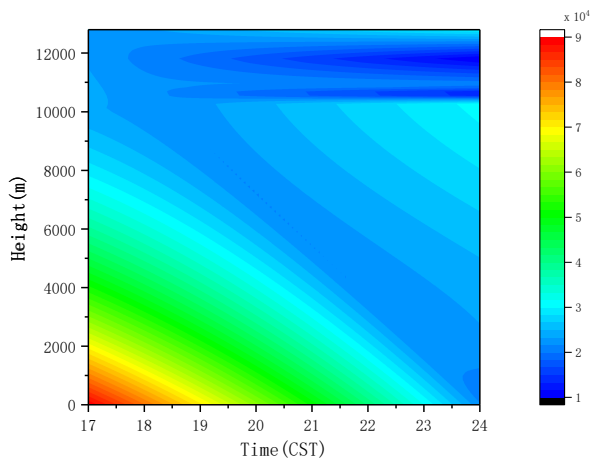

Figure 6. THI diagram of atmospheric aerosol distribution

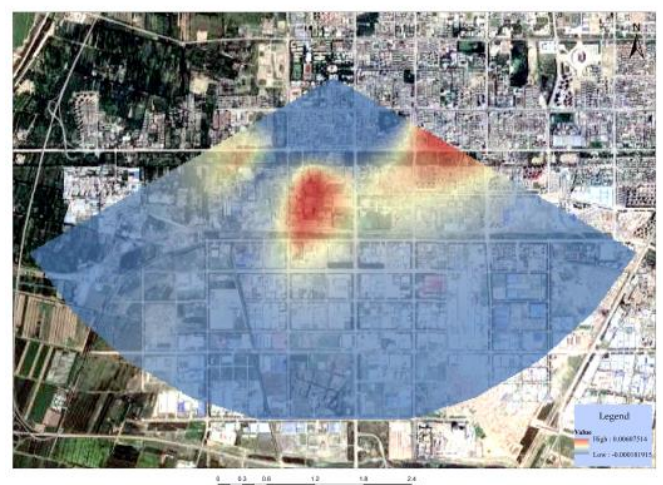

Figure 7. Slant scanning results

Figure 6 is the observation results of stagnation points from 17:00 to 24:00 on March 11, 2018. THI map was drawn with 100 sets of observation data. It can be seen from the figure that after 17:00, the area below $2 \mathrm{~km}$ has darker colors and the upper air has lighter colors. This is because human activities cause the near ground atmosphere to be turbidity. Around 24:00, the color 
is lighter in low altitude and darker in high altitude. From 5:00 to 24:00, the colors in the low altitude gradually become lighter and the colors in the high altitude gradually deepen. This is because the heat-island effect is reduced. The aerosols near the bottom are diluted, and the aerosol concentration gradually increases at upper air.

Figure 7 is the observation results of stagnation points at 21:20 on March 11, 2018. 10 groups of observation data are used for plotting. It can be clearly seen that the color is darker in the low altitude, it indicates that the concentration of aerosol particles is high and the absorption rate of aerosol particles to various wavelengths is high. With height increases, the color gradually becomes lighter, the aerosol concentration decreases, and the extinction coefficient of atmosphere also decreases. This is because the heat island effect generated by human activities, it leads to an aerosol layer near the ground, where the concentration of aerosol particles is larger and the absorption efficiency of light is greater. The maximum extinction coefficient can be obtained near ground. With the increase of height, the aerosol concentration is gradually decreased, and the corresponding extinction coefficient is also decreased.

By comparing Figure 6 and 7, the traditional THI diagram can only show the spatial-temporal evolution of aerosols by analyzing the distance. It cannot judge the pollution sources quickly. By using the new method, the temporal and spatial evolution of aerosols can be intuitively displayed on the map, and the source and formation mechanism of pollution sources can also be found. The traditional method requires more data to draw THI map, which increases the measurement time. It indicates that the traditional method has limitations. The new method improves the image quality and shows the evolution of aerosols in time and space with less data. The image is more intuitive and closer to reality than the traditional THI images. The drawing speed of a graph is faster than the traditional THI images, which can basically achieve real-time display.

From the above analysis, it can be seen that this method can quickly and accurately find out the pollution source when scanning at low altitude. When using slant scanning, it can effectively monitor the temporal and spatial distribution of aerosol concentration. The temporal and spatial evolution process of pollutants can be accurately and intuitively judged. So it can provide effective data support and prediction for environmental monitoring departments.

\section{CONCLUSION}

In this paper, a new method of plotting range-extinction coefficient diagram of aerosols using natural neighbourhood interpolation in ArcGIS is proposed. This method can quickly find the pollutants source and monitor the temporal and spatial evolution mechanism of aerosols.

This method can provide intuitive way to view aerosol distribution and provide the basis of decision-making for the weather bureau and environmental protection authorities. It can greatly improve work efficiency. In addition, the original observation data can be effectively tested by ArcGIS mapping. If there is an error in the original data, the monitor can quickly detect the anomaly and find the wrong data point, so as to quickly correct the data or overhaul the equipment.

\section{ACKNOWLEDGEMENTS}

This research was funded by the National Natural Science Foundation of China (Grant No. 61865001), the Ningxia Natural Science Foundation (Grant No. 2018AAC03103) and North MinZu University Innovation Project (Grant No. YCX18054).

\section{REFERENCES}

Althausen, D., Müller, D., Ansmann, A., 2000. Scanning 6-wavelength 11-channel aerosol lidar. Journal of Atmospheric and Oceanic Technology, 17(11), 1469-1482.

Chen, C., Wang, Z., Song, X., 2018. Development and observational studies of scanning aerosol lidar. Infrared and Laser Engineering, 47(12), 140-146.

Chen, X., Ji, M., Ning, F., 2016. Research on interpolation for fine forecast of meteorological grid. Geomatics and Spatial Information Technology, 39(10), 69-71.

Fan, X., Chen, H., Xia, X., 2013. Progress in observation studies of atmospheric aerosol radiative properties in China. Chinese Journal of Atmospheric Sciences, 37(02), 477-498.

Goldsmith, J.E.M., Forest, H.B., Scott, E.B., 1998. Turn -key Raman lidar for profiling atmospheric water vapor, clouds and aerosols. Applied Optics, 37(21), 4979-4990.

Liu, D., Tao, Z., Wu, D., 2013. Development of three-wavelength-raman-polarization lidar system and case study. Acta Optica Sinica, 33(2), 230-235.

Liu, H., Kong, X., Pan, H., 2018. The invention relates to the development and application of an aerosol monitoring lidar which can scan in all directions. Anhui Science and Technology, (03), 43-46.

Müller, D., Wandinger, U., Ansmann, A., 1999. Microphysical particle parameters from extinction and backscatter lidar data by inversion with regularization: simulation. Applied Optics, $38(12), 2358-2368$

Parsiani, H., Mendez, J., 2008. Aerosol size distribution using lidar data and a typical lidar assembly. Wseas Transactions on Systems, 7(11), 1218-1227.

Vakeva, M., Hameri, K., Puhakka, T., 2000. Effects of meteorological processes on aerosol particle size distribution in an urban background area. Journal of Geophysical Research: atmosphere, 105(D8), 9807-9821.

Wang, H., Cao, T., He, J., 2007. Characteristics study of atmospheric boundary layer by Mie scattering lidar in Chengdu. Journal of Atmospheric and Environmental Optics, (05), 340-344.

Xiao, C., 2017. The study and experiment of spatial data interpolation based on ArcGIS. Urban Geotechnical Investigation and Surveying, (06), 71-73.

Xu, Z., Pu, Q., Zou, B., 2017. A geographical information service system of urban air quality under Web mode. Science of Surveying and Mapping, 42(02), 47-52. 
You, H., Xing, Y., Ding, J., 2019. Research on DEM construction with different interpolation algorithms based on lidar data. Forest Engineering, 35(03), 20-25.

Young, M.N., Young, J.K., Byoung, C., 2007. Aerosol lidar ratio characteristics measured by a multi-wavelength Raman lidar system at Anmyeon Island, Korea. Atmospheric Research, 86(1), 76-87.

Zhang, X., Liu, L., Guo, H., 2018. Research on data conversion from shapefile to mapbox vector tile and its application. Geomatics World, 25(05), 109-113.

Zhong, Z., Liu, B., Fan, A., 2008. Two-wavelength Mie lidar with two receives. Journal of Atmospheric and Environmental Optics, 3(3), 173-178.

Zhao, D., Dang, D., Zhou, Z., 1988. Study on aerodynamic scale distribution of atmospheric aerosols in downtown Beijing in winter. Chinese Journal of Atmospheric Sciences, (02), 140-146.

Zhao, H., Hua, D., Di, H., 2015. Development of All time multi-wavelength lidar system and analysis of its signal to noise ratio. Chinese Journal of Lasers, 42(01), 284-291. 